\title{
Estudio para la implementación del manual de control interno caso (Gobierno Municipal del Cantón Puerto Quito, Provincia de Pichincha)
}

Study for the implementation of the case control manual (Municipal Government of the Canton of Puerto Quito, Province of Pichincha)

Ing. Fanny Graciela Egas Moreno ${ }^{1}$, Ing. Luz Marina Cifuentes ${ }^{2}$, Ing. Domitilo Toala Preciado ${ }^{3} \&$ Ing. Yadira Natalia Vergara Cuadros ${ }^{4}$

\begin{abstract}
DOI: https://doi.org/10.33262/cienciadigital.v9i2.372

His paper is based on the study for the implementation of an internal control manual for the accounting section of the cantonal government of Puerto Quito, Quito port canton, Pichincha Province, from January to June 2018. " And what poses as a problem, What will be the incidence of the study for the application of the implementation of an internal control manual for accounting section to reduce risks in the execution of activities aimed at meeting the objectives and in which the income and expenses applicable to the operations of the municipality, are they registered and adequately informed, with the purpose of preparing states and reliable financial reports? whose general objective is to provide the administration with an instrument that describes and unifies the minimum procedures and criteria that facilitate the evaluation of the internal control system by the internal control units, in order to promote their strengthening, providing retro feeder information for decision making in the municipality's management process, thus contributing to improving efficiency and effectiveness, which will benefit the interests of the Cantonal Government of Puerto Quito and the Accounting Section.
\end{abstract}

Keywords: Management control, administration, Municipal Government, resource evaluation.

\section{Resumen}

El presente trabajo se basa en el estudio para la implementación de un manual de control interno para la sección de contabilidad del Gobierno cantonal de Puerto

\footnotetext{
${ }^{1}$ Universidad Técnica Luis Vargas Torres de Esmeraldas, fanny.egas@utelvt.edu.ec

${ }^{2}$ Universidad Técnica Luis Vargas Torres de Esmeraldas, luz.cifuentes@utelvt.edu.ec

${ }^{3}$ Universidad Técnica Luis Vargas Torres de Esmeraldas, domitil2009@ hotmail.com

${ }^{4}$ Unidad Educativa Particular Juan Montalvo La Concordia, yadi.nat@hotmail.com
} 
Quito, Cantón Puerto Quito, Provincia de Pichincha, en el periodo de enero a junio del 2018”. Y que plantea como problema, ¿Cuál será la incidencia del estudio para la implementación de un manual de control interno para sección de contabilidad para disminuir riesgos en la ejecución de actividades encaminadas al cumplimiento de los objetivos y en que los ingresos y gastos aplicables a las operaciones del municipio, sean registradas e informadas adecuadamente, con el propósito de preparar estados e informes financieros confiables? cuyo objetivo general es dotar a la administración de un instrumento que describa y unifique los procedimientos y criterios mínimos que faciliten la evaluación del sistema de control interno por parte de las unidades de control interno, con el fin de fomentar su fortalecimiento, proporcionando información retro alimentadora para toma de decisiones en proceso de gestión de la municipalidad, contribuyendo así, a mejorar la eficiencia y efectividad, lo que redundara en beneficios de los intereses del Gobierno Cantonal de Puerto Quito y de las Sección de Contabilidad.

Palabras clave: Control de gestión, administración, Gobierno Municipal, evaluación de recursos.

\section{Introducción.}

El Municipio de Puerto Quito se encuentra funcionando administrativamente desde el mes de diciembre del año 1996, ha tenido cuatro administraciones en su vida institucional actualmente se encuentra liderada por una mujer la misma que es de la tendencia política del Movimiento Municipalista por la Integridad Nacional lista 24, cuenta con 120 personas que laboran en la institución dentro de estas se encuentran funcionarios, Empleados, y Trabajadores, (Prefectura de Pichincha , 2017), el Gobierno Cantonal de Puerto Quito en la sociedad política autónoma subordinada al orden jurídico Constitucional del Estado, su misión, es ser un Organismo Seccional auto suficiente, que provea de servicios básicos de excelente calidad tanto los residentes del cantón como de los turistas que lo visitan.

Para cumplir con la misión, el Gobierno Cantonal de Puerto Quito es una persona jurídica de derecho público, con patrimonio propio y con capacidad para realizar los actos jurídicos que fueren necesarios para el cumplimiento de sus fines, en la forma y condiciones que determinan la Constitución Política de la Republica y la ley orgánica de Régimen Municipal y más leyes conexas que tienen injerencias en su gestión. (OAS, 2013)

Los municipios, están involucrados necesariamente en los procesos de descentralizadores e importante sujeto a la modernización de su organización y como respuesta a ello, deberán asumir nuevas competencias y responsabilidades dentro del protagonismo que les corresponde como impulsadores y ejecutores del bienestar colectivo de sus localidades que representan. En este ámbito, están comprometidos a establecer políticas y estrategias para la mitigación de la pobreza, el desarrollo económico, la planificación participativa, la equidad de género de oportunidades ciudadanas generando paralelamente, la renovación y ejecución de proyectos y programas específicos de trabajo que transforme 
al municipio generadores del desarrollo social y económico (Carmona \& Barrios , 2007).

El alcance y frecuencia de las actividades de supervisión dependen de los riesgos a controlar y del grado de confianza que inspira a la dirección el proceso de control. La supervisión de los controles internos puede realizarse mediante actividades continúas incorporadas a los procesos empresariales y mediante evaluaciones separadas por parte de la dirección, de la función de auditorías internas o de personas independientes. Las actividades de supervisión continúan destinadas a comprobar la eficacia de los controles internos incluyen actividades periódicas de dirección y supervisión, comparaciones, y otras acciones de rutina. (Montiel, Montiel, \& Montiel, 2017)

El control interno amerita de un estudio concienzudo que permitirá definir su estrategia administrativa del sistema para conseguir la homogeneización de conceptos y formas tan necesarios para establecer modelos de información que puedan ser interpretados de forma similar y sirvan de elenco armonizador ante la verificación de la administración en la elaboración del proyecto que deben seguir determinados requisitos que garantice su eficiencia (Santillan, 2015).

Un sistema de control interno adecuado proporciona una relativa tranquilidad en el desarrollo de gestión de toda institución. La inexistencia o inadecuación del mismo, da lugar a riesgos importantes y complica erróneamente las actividades de la Dirección. Todo personal, especialmente el que cumple importantes funciones operativas o financieras, debe recibir y entender el mensaje de la alta dirección, de que las obligaciones en materia de control deben tomarse enserio. Asimismo, debe conocer su propio papel en el sistema de control interno, así como la forma en que sus actividades individuales se relacionan con el trabajo de los demás. Si no se conoce el sistema de control, los cometidos específicos y las obligaciones en el sistema, es probable que surjan problemas. Los empleados también deben conocer cómo sus actividades se relacionan con el trabajo de los demás. (Sierra , 2015)

El nuevo enfoque de control interno puede verse un poco riguroso, pero su actualidad, puede ser asimilada, de forma provechosa por la economía de las entidades. Sus cinco componentes son nuevos elementos que se aportan al sistema, se integran entre sí y se implementan de forma interrelacionada, influenciados por el estilo de dirección. Sirven para determinar si el sistema es eficaz. Marcan una diferencia con el enfoque tradicional de control interno dirigido al área financiera (Servicios de Rentas Internas , 2018). Dichos componentes de enmarcan en el sistema de gestión. Permiten prever los riesgos y tomar las medidas pertinentes para minimizar o eliminar su impacto en el cumplimiento de los objetivos organizacionales.

La presente investigación, consta de un Manual de control Interno, basado en lo previsto en el numeral 5 del artículo 61 de la Constitución de la República del Ecuador, como unos de los derechos de participación, ya que las ecuatorianas y ecuatorianos gozan de los derechos de fiscalizar los actos del poder público; en concordancia con el artículo 204 de la misma norma que contiene la función de la transparencia y control social, determinado que el pueblo es el mandante y el primer fiscalizador del poder público, en ejercicio de su derecho a la participación, respecto de todas las entidades y organismos del sector 
público, y de todas las personas naturales y jurídicas que presten servicios o desarrollen actividades de interés público. (Asamblea Nacional Del Ecuador , 2008)

La educación superior tiene la formación académica y profesional con visión científica y humanística, procurando la investigación científica y tecnológica, promoviendo la innovación, promoción, desarrollo y defunción de los saberes y culturas; la construcción de soluciones para los problemas del país, en relación con los objetivos del régimen de desarrollo, pues así lo ordena el artículo 350 de la Carta Magna. Específicamente la implementación de la auditoria interna en el Gobierno Cantonal de Puerto Quito, del Cantón del mismo nombre, provincia de Pichincha, fortalecerá la transparencia en el manejo de la organización del poder público, procurando cumplir a cabalidad los manuales de gestión de cada departamento dentro del organigrama estructural, así como también la simplificación de procedimientos, la pulcritud, diligencia en la utilización y distribución de los bienes y fondos públicos para alcanzar el bien común, con fin de toda sociedad civilizada. (Carmona \& Barrios , 2007)

El adecuado tratamiento y socialización de este procedimiento de auditoria no creara resistencia en los empleos y trabajadores del gobierno cantonal de puerto quito, pues este medio de control evitara futuras glosas, sanciones administrativa o pecuniarias por cualquier eventualidad que se presente en el desempeño de su función y fundamentalmente obtendremos la ansiedad transparencia y control social, que determinara una legitimidad de esta institución ante el pueblo como único mandante y soberano, eje del poder público. Siempre ha sido importante que los controles internos utilizados por instituciones públicas funcionen de manera correctas y la mejor forma es que esto sea evaluado periódica y eficazmente por un contrato público auditor. (Servicios de Rentas Internas , 2018)

Se presentarán las deficiencias que existan en los procesos administrativos de las instituciones públicas, sus causas, riesgos e implicaciones y se harán recomendaciones respectivas para cada hallazgo. Se hacen comentarios sobre los riesgos que podrían ocurrir si los procesos no son evaluados y monitoreados constantemente por el departamento de Auditoria Interna. Durante mucho tiempo los administradores se preocuparon solo de loa eficiencia de la maquina como medio para aumentar la productividad, pero esto no logro resolver el problema del aumento de la eficiencia de la organización. (Montiel, Montiel, \& Montiel, 2017).

Dado que el investigador labora en esta institución ha visto la necesidad de buscar estrategias para la Implementación de un Manual de Control interno, ya que no existe una seguridad razonable en la información para el cumplimiento de sus objetivos, es por ese motivo que se ha visto obligatorio a presentar un proyecto para dar solución al problema antes planteado (Sierra, 2015).

La inmediata aplicación del estudio del proyecto presentado la municipalidad planeará las actividades y contratará la ejecución del manual de control interno de acuerdo a las técnicas y normas establecidas en coordinación con las entidades que les puedan ayudar y los departamentos de la municipalidad a fines, y además se tomaran en cuenta a los deudores y acreedores. (Santillan, 2015). 


\section{Métodos y materiales}

Se trata de una investigación aplicada que busca resolver una situación problemática determinada y especifica que se presenta en el Municipio del cantón Puerto Quito. Se trata de una investigación de campo, que se desarrolla mediante un Diseño ProspectivoAnalítico, además bibliográfico - documental, descriptivo, exploratorio y explicativo que busca brindar una visión general de contexto del problema se aplicó una encuesta a una muestra demostrada en la siguiente tabla.

Tabla 1. Departamento de personal

\begin{tabular}{|l|c|c|c|}
\hline PERSONAL & DE CARRERA & CONTRATADOS & TOTAL \\
\hline Empleados & 17 & 6 & 23 \\
\hline Trabajadores & 38 & 9 & 47 \\
\hline Total & 55 & 15 & 70 \\
\hline
\end{tabular}

Elaborado por: Grupo investigador

\section{Análisis de resultados}

Tabla 2. Diagrama: departamento de contabilidad

\begin{tabular}{|c|c|c|c|c|c|c|c|}
\hline \multirow{2}{*}{$\mathbf{N}^{\circ}$} & \multirow{2}{*}{ CUESTIONARIO } & \multicolumn{3}{|c|}{ RESPUESTAS } & \multicolumn{2}{|c|}{ VALOR } & \multirow{2}{*}{ OBSERVACION } \\
\hline & & SI & NO & $\mathrm{NC}$ & POND & CALF & \\
\hline 1 & $\begin{array}{l}\text { Se efectúan tomas } \\
\text { físicas de los } \\
\text { activos fijos por lo } \\
\text { menos una vez al } \\
\text { año }\end{array}$ & & $X$ & & 6 & 4 & Cada año \\
\hline 2 & $\begin{array}{l}\text { Los registros de los } \\
\text { activos fijos se } \\
\text { encuentran } \\
\text { actualizados }\end{array}$ & $\mathrm{X}$ & & & 8 & 8 & Cada año \\
\hline 3 & $\begin{array}{l}\text { Los activos fijos } \\
\text { están } \\
\text { perfectamente } \\
\text { codificados para su } \\
\text { identificación }\end{array}$ & & & $X$ & 7 & 5 & Departamento \\
\hline 4 & $\begin{array}{l}\text { Se controlan } \\
\text { adecuadamente los } \\
\text { movimientos } \\
\text { internos de los } \\
\text { activos fijos } \\
\text { institucionales }\end{array}$ & $\mathrm{X}$ & & & 8 & 6 & Contabilidad \\
\hline
\end{tabular}




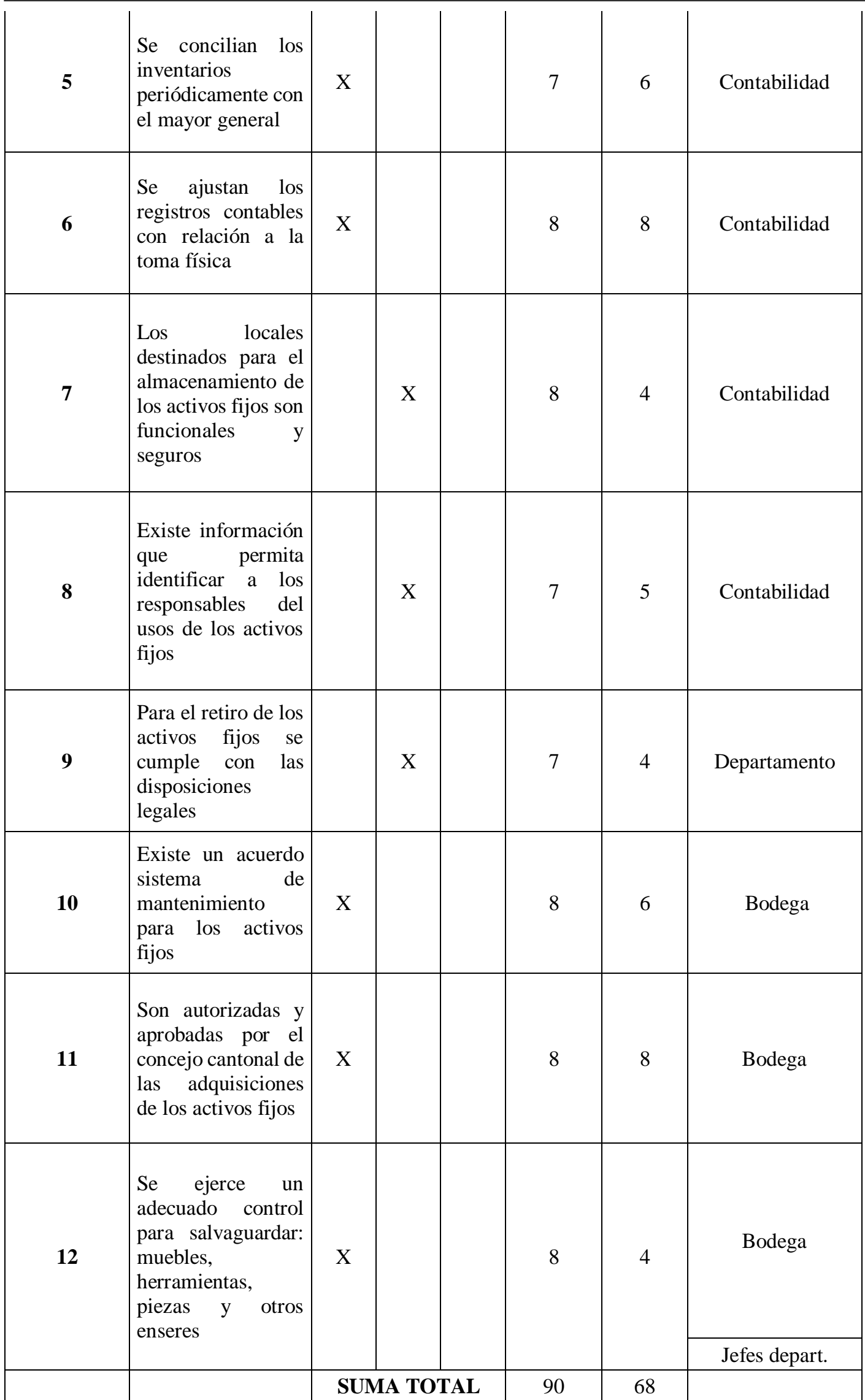

Elaborado por: Grupo investigador 
$>$ Ponderación total

$$
\text { (P.T. })=90
$$

Calificación total

(C.T. $)=68$

$>$ Calificación porcentual

(C.P.) $=100 \%$

$$
\mathrm{CP}=\frac{C T \times 100}{P T}=\frac{68 \times 100}{90}=75 \%
$$

Tabla 3. Ponderación de los activos fijos

\begin{tabular}{|c|c|c|c|}
\hline Riesgo y eficiencia & 1 & 2 & 3 \\
\hline Confianza ò solidez & $15 \%$ al $50 \%$ & $51 \%$ al $75 \%$ & $76 \%$ al $100 \%$ \\
\hline RESULTADOS & \multicolumn{3}{|c|}{$75 \%$} \\
\hline
\end{tabular}

Elaborado por: Grupo investigador

Gráfico 1. Activos fijos

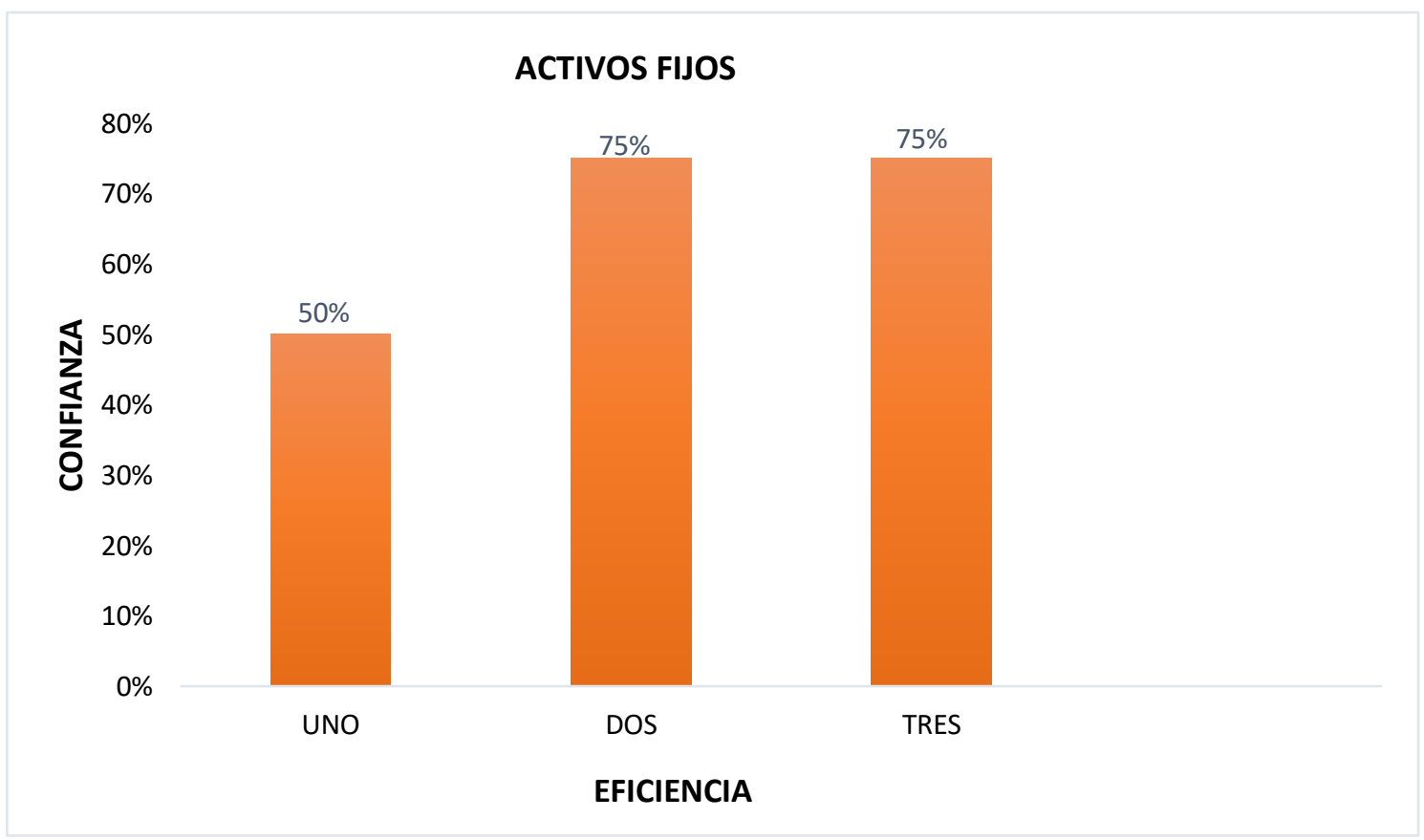

Como producto del estudio y análisis del cuestionario de Activos Fijos. La prueba de cumplimiento es departamental: El Departamento contable, podría decirse que por lo general los registros y tomas físicas se lo realizan por lo menos una vez al año, según las normas establecidas por la Contraloría. El nivel de confianza es del 75\%, la ponderación es mayor a la calificación por los procedimientos que utiliza la institución en el tratamiento de los activos fijos por lo que el factor de solidez esta en nivel dos de riesgo y eficiencia. La responsabilidad del departamento de contabilidad en lo que se refiera a la adquisición de productos que son controlados y registrados de acuerdo como lo exige la Norma Contable y el reglamento Interno del Municipio para que estos bienes sean utilizados de manera eficiente en las comunidades. Se recomendaría una mayor eficiencia en el mantenimiento de los Activos Fijos para que su utilización sea óptima en las actividades de las comunidades, considerando que el uso de herramientas y otros necesarios para las prácticas contables que realiza el Municipio son prioritarios. 
Evaluación de los inventarios.

Tabla 4. Diagrama: departamento de contabilidad

\begin{tabular}{|c|c|c|c|c|c|c|c|}
\hline \multirow{2}{*}{$\mathbf{N}^{\circ}$} & \multirow{2}{*}{ CUESTIONARIO } & \multicolumn{3}{|c|}{ RESPUESTAS } & \multicolumn{2}{|c|}{ VALOR } & \multirow{2}{*}{$\begin{array}{c}\text { OBSERVACION } \\
\text { Prueba de cumplimiento }\end{array}$} \\
\hline & & SI & NO & $\mathrm{NC}$ & POND & CALF & \\
\hline 1 & $\begin{array}{l}\text { Tienen } \\
\text { procedimientos para } \\
\text { determinar las } \\
\text { existencias de posibles } \\
\text { artículos, muebles, } \\
\text { herramientas dañadas }\end{array}$ & $\mathrm{X}$ & & & 8 & 7 & Contabilidad \\
\hline 2 & $\begin{array}{l}\text { Son adecuados los } \\
\text { procedimientos para la } \\
\text { toma física de los } \\
\text { inventarios }\end{array}$ & & $\mathrm{X}$ & & 10 & 6 & Bodega \\
\hline 3 & $\begin{array}{l}\text { Se efectúan } \\
\text { adecuadamente los } \\
\text { procedimientos para la } \\
\text { adquisición, } \\
\text { autorización, custodia } \\
\text { y registros contables } \\
\text { de los inventarios. }\end{array}$ & $\mathrm{X}$ & & & 8 & 8 & Contabilidad \\
\hline 4 & $\begin{array}{lr}\text { Se lleva control } \\
\text { contable de las } \\
\text { existencias de los } \\
\text { bienes del Municipio } \\
\text { que informen las } \\
\text { características } \\
\text { valores que respalden } \\
\text { los saldos del mayor } \\
\text { general }\end{array}$ & $\mathrm{X}$ & & & 10 & 6 & Contabilidad \\
\hline 5 & $\begin{array}{l}\text { Se mantiene un acceso } \\
\text { limitado a los lugares } \\
\text { donde se encuentran } \\
\text { las existencias }\end{array}$ & & & $\mathrm{X}$ & 8 & 9 & Bodega \\
\hline 6 & $\begin{array}{lr}\text { Se lleva un registro } \\
\text { codificado de los } \\
\text { bienes del municipio } \\
\text { que permitan su } \\
\text { identificación y los } \\
\text { responsables de } \\
\text { utilización }\end{array}$ & $\mathrm{X}$ & & & 10 & 9 & Bodega \\
\hline
\end{tabular}




\begin{tabular}{|c|c|c|c|c|c|c|}
\hline 7 & $\begin{array}{lr}\text { Se mantiene stock } \\
\text { mínimos y máximos } \\
\text { para efectuar las } \\
\text { adquisiciones } \\
\text { consumos } \\
\text { agropecuarios }\end{array}$ & $\mathrm{x}$ & & 10 & 9 & Contabilidad \\
\hline 8 & $\begin{array}{l}\text { Se lleva a cabo una } \\
\text { comparación periódica } \\
\text { de los registros con los } \\
\text { saldos de la cuenta del } \\
\text { mayor }\end{array}$ & & $\mathrm{X}$ & 6 & 5 & Departamento bodega \\
\hline 9 & $\begin{array}{l}\text { Se cumplen los } \\
\text { procedimientos de tres } \\
\text { preformas para la } \\
\text { adquisición de bienes } \\
\text { e insumos }\end{array}$ & & $\mathrm{x}$ & 8 & 6 & Departamento \\
\hline 10 & 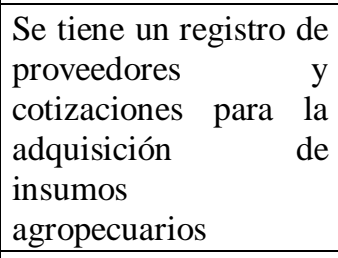 & $\mathrm{X}$ & & 10 & 8 & Contabilidad \\
\hline 11 & $\begin{array}{l}\text { En el Concejo } \\
\text { Cantonal cumple con } \\
\text { todas las normas y } \\
\text { procedimientos que } \\
\text { exige la contraloría } \\
\text { para la adquisición de } \\
\text { bienes para el } \\
\text { municipio }\end{array}$ & $\mathrm{X}$ & & 8 & 7 & Contabilidad \\
\hline 12 & $\begin{array}{l}\text { Existe un control de la } \\
\text { documentación que } \\
\text { justifique la entrada y } \\
\text { salida de los } \\
\text { inventarios } \\
\end{array}$ & $\mathrm{x}$ & & 10 & 9 & Jefes depart. \\
\hline & & \multicolumn{2}{|c|}{ SUMA TOTAL } & 106 & 89 & \\
\hline
\end{tabular}

Elaborado por: Grupo investigador

\section{Valoración:}

Ponderación total

$$
(\text { P.T. })=106
$$

$>$ Calificación total

(C.T. $)=89$

$>$ Calificación porcentual

$$
\mathrm{CP}=\frac{C T \times 100}{P T}=\frac{89 \times 100}{106}=84 \%
$$


Tabla 5. Ponderación de la evaluación de inventarios

\begin{tabular}{|c|c|c|c|}
\hline Riesgo y eficiencia & 1 & 2 & 3 \\
\hline Confianza ò solidez & $15 \%$ al $50 \%$ & $51 \%$ al $75 \%$ & $76 \%$ al $100 \%$ \\
\hline RESULTADOS & \multicolumn{3}{|c|}{$84 \%$} \\
\hline
\end{tabular}

Elaborado por: Grupo investigador.

Gráfico. 2.Evaluación de inventarios

EVALUACIÓN DE INVENTARIOS

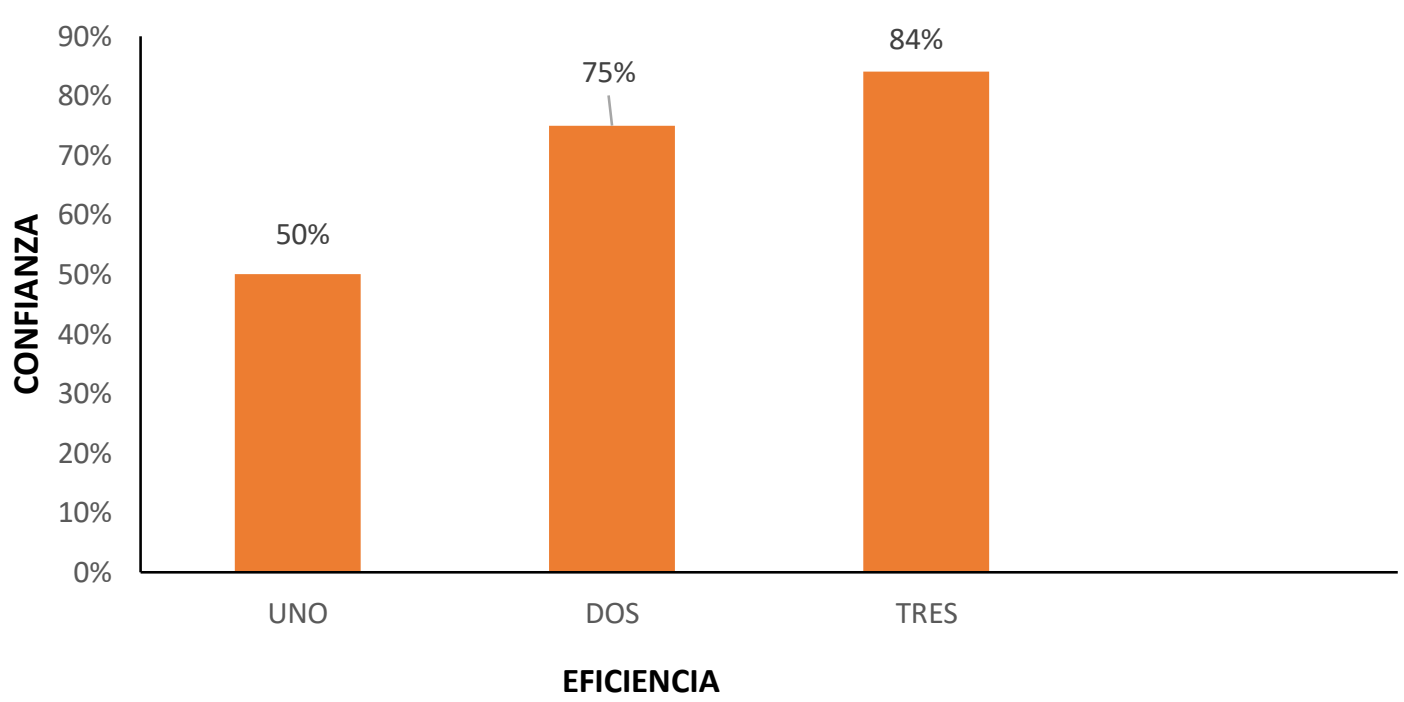

La evaluación de los inventarios del municipio del cantón Puerto Quito se estableció que la responsabilidad está a cargo del departamento de la contabilidad en relación con bodega y requisiciones. Los valores de la ponderación nos dan significativamente un resultado mayor en relación con la calificación y su evaluación de confianza es del $84 \%$ que se encuentra en el segundo nivel. Esto nos demuestra que la solidez en los inventarios no es la óptima, siendo la falla en la adquisición de insumos agropecuarios para su utilización oportuna en las actividades prácticas de las comunidades. Podría existir otra falla al realizar los pedidos o adquisiciones por parte de los jefes departamentales o también, al cumplir los tramites que se exigen para la adquisición de los insumos. El Municipio como Institución pública, deberá optimizar el control contable señala en las disposiciones legales y se deberá cumplir con las normas establecidas en la Ley orgánica de la administración financiera, que permiten el manejo, custodia, salvaguarda y mantenimiento de los bienes físicos, estructurales, equipos e insumos que tiene el municipio.

Evaluación de las especies valoradas. 
Tabla 6. Departamento de contabilidad

\begin{tabular}{|c|c|c|c|c|c|c|c|}
\hline \multirow{2}{*}{$\mathbf{N}^{\circ}$} & \multirow{2}{*}{ CUESTIONARIO } & \multicolumn{3}{|c|}{ RESPUESTAS } & \multicolumn{2}{|c|}{ VALOR } & \multirow{2}{*}{$\begin{array}{c}\text { OBSERVACION } \\
\text { Prueba de cumplimiento }\end{array}$} \\
\hline & & SI & NO & $\mathbf{N C}$ & POND & CALF & \\
\hline $\mathbf{1}$ & $\begin{array}{l}\text { La emisión de } \\
\text { especies valoradas es } \\
\text { autorizadas por la } \\
\text { autoridad competente, } \\
\text { Concejo Cantonal }\end{array}$ & $\mathrm{X}$ & & & 8 & 8 & Tesoreria \\
\hline 2 & $\begin{array}{lr}\text { Existe un control } \\
\text { sobre la emisión de } \\
\text { especies } & \text { valoradas } \\
\text { facturas y } & \text { recibos. } \\
\text { Según su } & \text { secuencia } \\
\text { cronológica } & \text { y } \\
\text { numérica } & \end{array}$ & & & $X$ & 8 & 6 & Departamento \\
\hline 3 & \begin{tabular}{lr}
\multicolumn{2}{l}{ Las especies valoradas } \\
canceladas & son \\
registradas & según \\
procedimientos & y \\
contabilizadas &
\end{tabular} & $\mathrm{X}$ & & & 8 & 6 & Contabilidad \\
\hline 4 & $\begin{array}{l}\text { Se encuentran las } \\
\text { especies valoradas } \\
\text { debidamente } \\
\text { salvaguardadas }\end{array}$ & $\mathrm{X}$ & & & 10 & 7 & Contabilidad \\
\hline 5 & $\begin{array}{lr}\text { Las personas } & \text { que } \\
\text { trabajan en el área } \\
\text { financiera } & \text { en } \\
\text { funciones } & \text { de } \\
\text { recaudación } & \text { son } \\
\text { caucionadas } & \end{array}$ & & $\mathrm{X}$ & & 8 & 5 & Contabilidad \\
\hline 6 & $\begin{array}{l}\text { Se realiza una } \\
\text { verificación por un } \\
\text { miembro del concejo } \\
\text { cantonal que no sea la } \\
\text { Tesorera }\end{array}$ & $\mathrm{X}$ & & & 10 & 8 & Funcionarios de carrera \\
\hline 7 & $\begin{array}{l}\text { La recaudación de los } \\
\text { ingresos son } \\
\text { depositados en le } \\
\text { cuenta del municipio } \\
\text { de manera inmediata y } \\
\text { contabilizado con los } \\
\text { debidos soportes }\end{array}$ & $X$ & & & 9 & 8 & Contabilidad \\
\hline 8 & $\begin{array}{l}\text { Se encuentran } \\
\text { registrados } \\
\text { contabilizados los } \\
\text { ingresos por concepto } \\
\text { de especies valoradas }\end{array}$ & $\mathrm{X}$ & & & 8 & 5 & Contabilidad \\
\hline
\end{tabular}




\begin{tabular}{|c|c|c|c|c|c|c|}
\hline 9 & $\begin{array}{l}\text { Cada que tiempo } \\
\text { cumple la contraloría, } \\
\text { revisa e informa sobre } \\
\text { las especies valoradas } \\
\text { e ingresos }\end{array}$ & & $\mathrm{X}$ & 8 & 5 & Departamento \\
\hline 10 & $\begin{array}{l}\text { El consejo cantonal } \\
\text { conoce y autoriza la } \\
\text { emisión e ingresos por } \\
\text { especies valoradas }\end{array}$ & $\mathrm{X}$ & & 8 & 5 & Contraloría cada año \\
\hline & & \multicolumn{2}{|c|}{ SUMA TOTAL } & 85 & 63 & \\
\hline
\end{tabular}

Elaborado por: Grupo investigador

\section{Valoración:}

Ponderación total

$$
(\mathrm{P} . \mathrm{T} .)=85
$$

$>$ Calificación total

(C.T.) $=63$

$>$ Calificación porcentual

$$
\text { (C.P.) }=100 \%
$$

$$
\mathrm{CP}=\frac{C T \times 100}{P T}=\frac{63 \times 100}{85}=74 \%
$$

Tabla 7. Ponderación de las especies valoradas

\begin{tabular}{|c|c|c|c|}
\hline Riesgo y eficiencia & 1 & 2 & 3 \\
\hline Confianza ò solidez & $15 \%$ al $50 \%$ & $51 \%$ al $75 \%$ & $76 \%$ al $100 \%$ \\
\hline RESULTADOS & \multicolumn{3}{|c|}{$74 \%$} \\
\hline
\end{tabular}

Elaborado por: Grupo investigador

Gráfico. 3. Especies valoradas

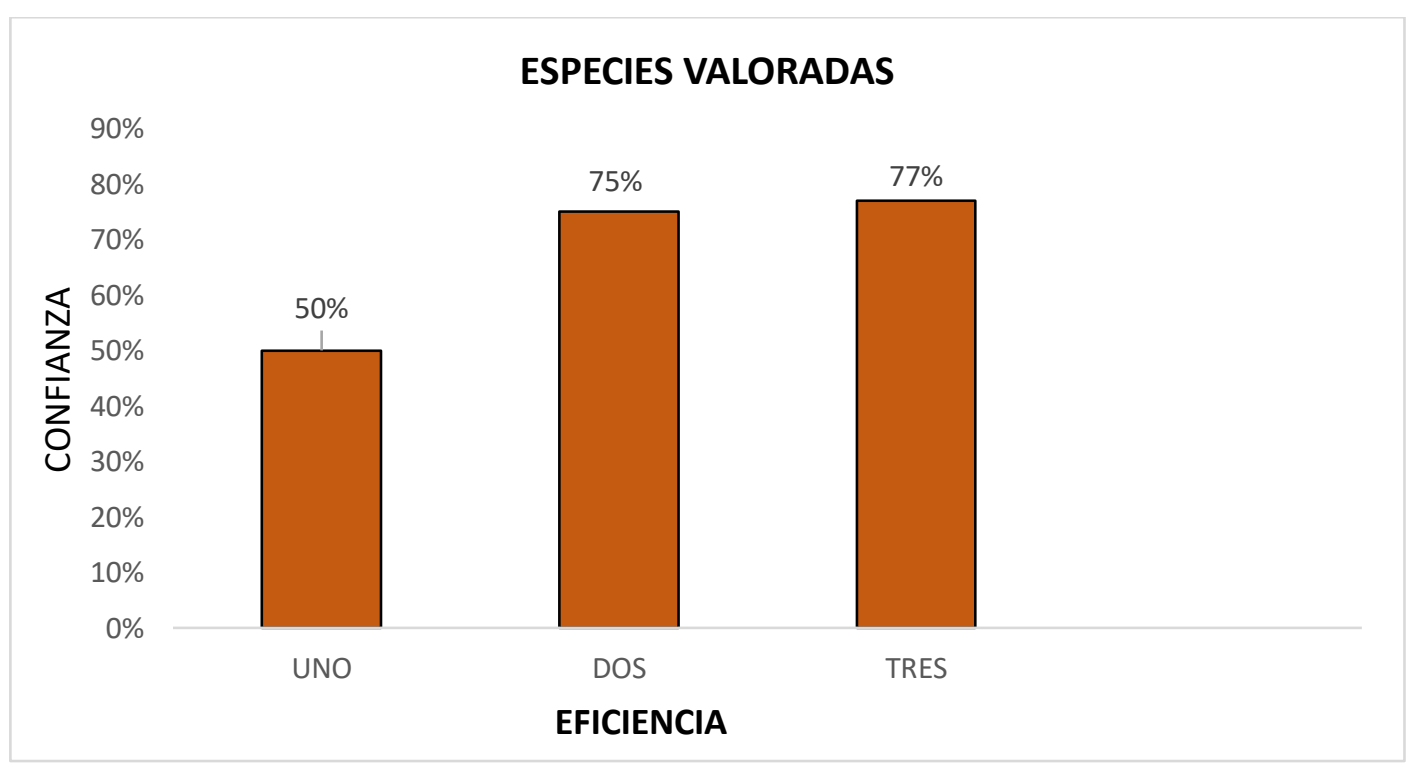

El Municipio del cantón Puerto Quito, realiza cobros mediante la venta de Especies 
Valoradas como: permisos, derechos solicitudes y uso de insumos, materiales y herramientas a los estudiantes que tiene el Municipio, por lo que la Contraloría General del Estado debe aplicar sus especies e ingresos monetarios causados por este tipo de actividad o servicios; de igual manera relacionar mediante informes desde la Tesorería al Departamentos y este a su vez al Consejo Cantonal para conocimiento del usos de estos valores depositados en la cuenta bancaria que mantiene el Municipio en sus haberes. Al analizar el cuestionario de Especies Valoradas el nivel de confianza y solidez es del 74\% que es el resultado de la ponderación mayor a la calificación, que recae directamente al departamento de contabilidad a través de la Tesorería. Además, nos muestra una baja significativa la calificación debido a la falta de control por parte de las autoridades de la institución, sin embargo, se cumple a medias con los procedimientos para la emisión y adquisición de Especies valoradas.

\section{Evaluación de los ingresos.}

Tabla 8. Departamentos de contabilidad

\begin{tabular}{|c|c|c|c|c|c|c|c|}
\hline \multirow{2}{*}{$\mathbf{N}^{\circ}$} & \multirow{2}{*}{ CUESTIONARIO } & \multicolumn{3}{|c|}{ RESPUESTAS } & \multicolumn{2}{|c|}{ VALOR } & \multirow{2}{*}{$\begin{array}{c}\text { OBSERVACION } \\
\text { Prueba de cumplimiento } \\
\end{array}$} \\
\hline & & SI & NO & $\mathrm{NC}$ & POND & CALF & \\
\hline 1 & $\begin{array}{l}\text { Existen formularios o } \\
\text { registros para los } \\
\text { ingresos debidamente } \\
\text { impresos y pre- } \\
\text { numerados }\end{array}$ & $\mathrm{X}$ & & & 8 & 8 & Contabilidad \\
\hline 2 & $\begin{array}{lr}\text { Los registros } \\
\text { contables están } \\
\text { debidamente } \\
\text { detallados para el } \\
\text { control de los egresos }\end{array}$ & $\mathrm{x}$ & & & 8 & 8 & Contabilidad \\
\hline 3 & $\begin{array}{lr}\text { Se } & \text { archivan } \\
\text { adecuadamente } & \text { los } \\
\text { comprobantes } & \text { de } \\
\text { ingresos. } & \end{array}$ & & & $\mathrm{x}$ & 8 & 8 & Contabilidad \\
\hline 4 & $\begin{array}{lrr}\text { El registro de } & \text { los } \\
\text { ingresos } & & \text { se } \\
\text { encuentran } & & \\
\text { actualizados } & & \end{array}$ & $\mathrm{X}$ & & & 10 & 6 & Contabilidad \\
\hline 5 & $\begin{array}{l}\text { Las cuentas bancarias } \\
\text { están registradas, } \\
\text { según las partidas de } \\
\text { asignación de fondos } \\
\text { del Estado }\end{array}$ & & & $\mathrm{x}$ & 8 & 4 & Contabilidad \\
\hline 6 & $\begin{array}{lr}\text { Se encuentran } \\
\text { perfectamente } \\
\text { legalizados } \\
\text { comprobantes } \\
\text { ingresos. }\end{array}$ & $\mathrm{X}$ & & & 10 & 6 & Contabilidad \\
\hline
\end{tabular}




\begin{tabular}{|c|c|c|c|c|c|c|}
\hline 7 & $\begin{array}{lr}\text { Se } & \text { controla } \\
\text { numéricamente } & \text { los } \\
\text { comprobantes } & \text { de } \\
\text { ingresos a las cuentas } \\
\text { bancarias } \\
\text { Municipio }\end{array}$ & $\mathrm{X}$ & & 10 & 6 & Contabilidad \\
\hline 8 & \begin{tabular}{lr}
\multicolumn{2}{l}{ Existen control de los } \\
desembolsos & e \\
ingresos en & efectivo \\
de las & cuentas \\
bancarias & del \\
Municipio &
\end{tabular} & & $\mathrm{x}$ & 8 & 6 & Contabilidad \\
\hline 9 & $\begin{array}{lr}\text { Existen control de } \\
\text { cheques emitidos por } \\
\text { el municipio ya } & \text { sea } \\
\text { por concepto } & \text { de } \\
\text { adquisición o } & \text { por } \\
\text { pagos a terceros } & \end{array}$ & $\mathrm{x}$ & & 8 & 7 & Tesorería \\
\hline 10 & $\begin{array}{l}\text { Los depósitos por } \\
\text { especies valoradas } \\
\text { son realizadas } \\
\text { oportunamente }\end{array}$ & $\mathrm{X}$ & & 10 & 9 & Tesorería o departamento \\
\hline & & \multicolumn{2}{|c|}{ SUMA TOTAL } & 88 & 68 & \\
\hline
\end{tabular}

Elaborado por: Grupo investigador

\section{Valoración:}

Ponderación total

$$
(\text { P.T. })=88
$$

Calificación total

(C.T. $)=68$

$>$ Calificación porcentual

(C.P. $)=100 \%$

$$
\mathrm{CP}=\frac{C T \times 100}{P T}=\frac{68 \times 100}{88}=77 \%
$$

Tabla 9. Ponderación de los ingresos

\begin{tabular}{|c|c|c|c|}
\hline Riesgo y eficiencia & 1 & 2 & 3 \\
\hline Confianza ò solidez & $15 \%$ al $50 \%$ & $51 \%$ al $75 \%$ & $76 \%$ al $100 \%$ \\
\hline RESULTADOS & \multicolumn{3}{|c|}{$77 \%$} \\
\hline
\end{tabular}

Elaborado por: Grupo investigador.

Gráfico 4. Ingresos 
INGRESOS

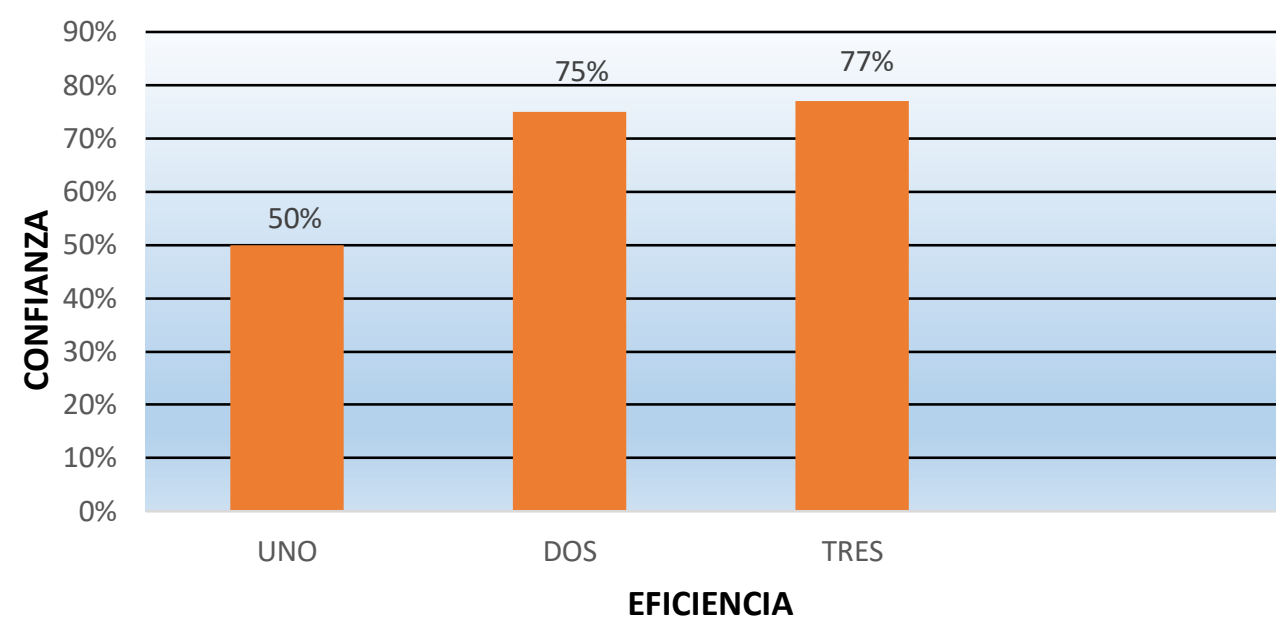

Según la evaluación de los ingresos del Municipio del Cantón Puerto Quito. Los responsables de la ejecución del presupuesto deben efectuar seguimientos y verificaciones permanentes para dar cumplimiento a lo estipulado por la ley.

Los ingresos deben ser registrados mensualmente según las asignaciones del presupuesto que envía el estado a las cuentas bancarias que tiene el municipio en la banca privada y debe ser registrada y documentada, con los respectivos justificativos que establece la Ley para la transferencia de Fondos a las cuentas de la institución. El resultado del análisis del cuestionario respecto a los ingresos, la prueba de cumplimiento es responsabilidad del departamento de contabilidad y Tesorería. La ponderación nos muestra una diferencia de 28 puntos dando un resultado en el segundo nivel de confianza y solidez y aplicando la matriz, nos da un valor del 77\%. Por lo que las autoridades del municipio deben exigir ue cumpla con las disociaciones de Ley que están sujetos al control de la Contraloría y Departamento de Contabilidad, exigir que cumpla con las disposiciones de Ley en la transferencia de fondos del Estado a las cuentas bancarias de la institución

\section{Evaluación de los egresos.}

Tabla 10. Departamentos de contabilidad

\begin{tabular}{|c|c|c|c|c|c|c|c|}
\hline \multirow{2}{*}{$\mathbf{N}^{\circ}$} & \multirow{2}{*}{ CUESTIONARIO } & \multicolumn{3}{|c|}{ RESPUESTAS } & \multicolumn{2}{|c|}{ VALOR } & OBSERVACION \\
\hline & & SI & NO & $\mathrm{NC}$ & POND & CALF & CONTABILIDAD \\
\hline 1 & $\begin{array}{l}\mathrm{Se} \text { efectúan } \\
\text { desembolsos } \\
\text { mediante cheques } \\
\text { nominativos y } \\
\text { autorizados }\end{array}$ & $\mathrm{X}$ & & & 8 & 8 & TESORERIA \\
\hline 2 & $\begin{array}{l}\text { Se lleva un registro } \\
\text { contable para el } \\
\text { control de los } \\
\text { egresos }\end{array}$ & $\mathrm{X}$ & & & 8 & 8 & TESORERIA \\
\hline
\end{tabular}




\begin{tabular}{|c|c|c|c|c|c|c|c|}
\hline 3 & $\begin{array}{l}\text { Los comprobantes } \\
\text { de egresos y } \\
\text { cheques cancelados } \\
\text { son registrados }\end{array}$ & $X$ & & & 6 & 8 & TESORERIA \\
\hline 4 & $\begin{array}{l}\text { Los desembolsos } \\
\text { cuentan con la } \\
\text { debida } \\
\text { documentación } \\
\text { justificada }\end{array}$ & $\mathrm{X}$ & & & 10 & 9 & TESORERIA \\
\hline 5 & $\begin{array}{l}\text { Los cheques } \\
\text { emitidos siguen el } \\
\text { orden cronológico } \\
\text { numérico } \\
\text { ascendente. }\end{array}$ & $X$ & & & 10 & 9 & TESORERIA \\
\hline \multirow{4}{*}{6} & $\begin{array}{l}\text { Existen prohibición } \\
\text { para: }\end{array}$ & & \multirow{4}{*}{$\mathrm{X}$} & & \multirow{4}{*}{8} & \multirow{4}{*}{7} & \multirow{4}{*}{ TESORERIA } \\
\hline & $\begin{array}{l}\text { a) Emitir cheques } \\
\text { al portador }\end{array}$ & & & & & & \\
\hline & $\begin{array}{l}\text { b) Firmar cheques } \\
\text { por fechadores. }\end{array}$ & & & & & & \\
\hline & $\begin{array}{l}\text { Firmar cheques en } \\
\text { blanco }\end{array}$ & & & & & & \\
\hline 7 & $\begin{array}{lr}\text { Se verifica la la } & \text { disponibilidad de } \\
\text { las cuentas } \\
\text { las bancarias del } \\
\text { Municipio, antes de } \\
\text { girar los cheques. }\end{array}$ & $X$ & & & 10 & 5 & TESORERIA \\
\hline 8 & $\begin{array}{l}\text { Las conciliaciones } \\
\text { bancarias son } \\
\text { efectuadas por la } \\
\text { recolectora, rector }\end{array}$ & & & $\mathrm{X}$ & 8 & 4 & CONTABILIDAD \\
\hline 9 & $\begin{array}{l}\text { Los egresos en } \\
\text { Gerencia están } \\
\text { debidamente } \\
\text { documentados y } \\
\text { justificados para } \\
\text { control por parte de } \\
\text { la contraloría. }\end{array}$ & $\mathrm{X}$ & & & 8 & 5 & CONTABILIDAD \\
\hline 10 & $\begin{array}{l}\mathrm{Se} \text { realiza } \\
\text { conciliaciones } \\
\text { bancarias } \\
\text { mensuales de las } \\
\text { cuentas que tiene el } \\
\text { Municipio }\end{array}$ & $\mathrm{X}$ & & & 10 & 7 & CONTABILIDAD \\
\hline & & SL & $\mathrm{TC}$ & $\mathbf{A L}$ & 86 & 70 & \\
\hline
\end{tabular}


Elaborado por: Grupo investigador

\section{Valoración:}

Ponderación total

$$
\text { (P.T. })=86
$$

$>$ Calificación total

(C.T.) $=70$

$>$ Calificación porcentual

$$
\text { (C.P.) }=100 \%
$$

$$
\mathrm{CP}=\frac{C T \times 100}{P T}=\frac{70 \times 100}{86}=81 \%
$$

Tabla 11. Ponderación de los egresos

\begin{tabular}{|c|c|c|c|}
\hline Riesgo y eficiencia & 1 & 2 & 3 \\
\hline Confianza ò solidez & $15 \%$ al $50 \%$ & $51 \%$ al $75 \%$ & $76 \%$ al $100 \%$ \\
\hline RESULTADOS & \multicolumn{3}{|c|}{$81 \%$} \\
\hline
\end{tabular}

Elaborado por: Grupo investigador

Gráfico. 5. Egresos

\section{EGRESOS}

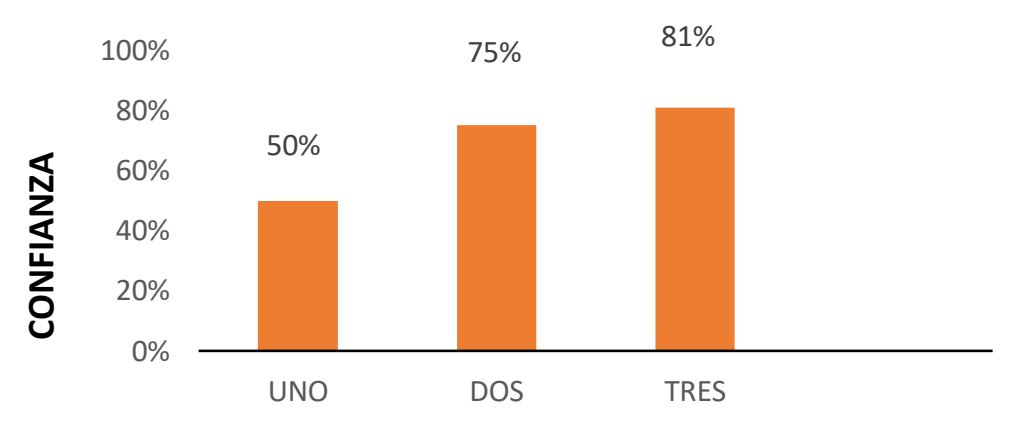

EFICIENCIA

El departamento de contabilidad y tesorería es la parte operativa para cumplir con los trámites y documentación necesaria para el manejo de las cuentas que tiene el municipio. Siendo la alcaldesa del municipio como autoridad la responsable de aprobar con su firma todo pago, como son los gastos operacionales o gastos generales, contratación o pagos a terceros debe comprobar que la tesorería cumpla con las disposiciones legales contempladas en la Ley y Reglamento del Municipio del cantón Puerto Quito. Las cuentas por ingresos y egresos están relacionadas contablemente y son el componente más importante del presupuesto de la institución. Por lo tanto, la alcaldesa debe exigir que los gastos sean controlados y registrados, para garantizar durante el año escolar la utilización de los recursos básicos, adquisiciones de bienes e insumos que necesita el municipio debido a su carácter de institución pública. El cuestionario de los egresos nos demuestra que se sitúa en el nivel segundo por lo tanto la confianza y solidez cae en $81 \%$ estos significan que se debe optimizar al departamento de contabilidad y tesorería con el departamento para dar eficiencia en el cumplimiento de las disposiciones de Ley que se 
tiene para la utilización de los recursos económicos que cuenta la Institución.

\section{Valoración:}

Ponderación total

$($ P.T. $)=455$

$>$ Calificación total

(C.T.) $=358$

Calificación porcentual

(C.P.) $=100 \%$

$$
\mathrm{CP}=\frac{C T \times 100}{P T}=\frac{358 \times 100}{455}=78 \%
$$

Tabla 12. Ponderación al personal

\begin{tabular}{|c|c|c|c|}
\hline Riesgo y eficiencia & 1 & 2 & 3 \\
\hline Confianza ò solidez & $15 \%$ al $50 \%$ & $51 \%$ al $75 \%$ & $76 \%$ al $100 \%$ \\
\hline RESULTADOS & \multicolumn{3}{|c|}{$78 \%$} \\
\hline
\end{tabular}

Elaborado por: Grupo investigador

Gráfico. 6. Personal

PERSONAL

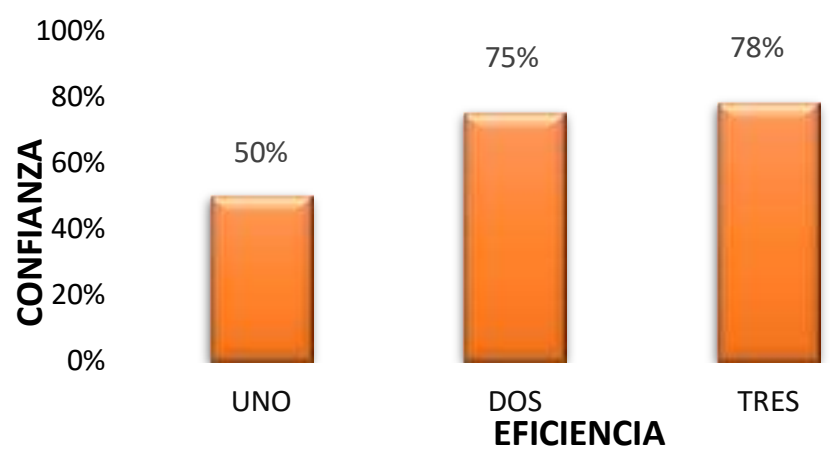

En el rubro de especies valoradas se llegó a determinar que el departamento de tesorería no está realizando la contabilización en forma oportuna por concepto de ventas de dichas especies y de igual manera las conciliaciones bancarias no se las hace a su debido tiempo ni tampoco hay evidencia documentada de que se haya hecho las constataciones físicas de las especies valoradas en existencia.

\section{Conclusiones.}

- En el departamento de tesorería no hay un adecuado control y organización de la contabilización de las especies valoradas, afectando de manera diferente a las conciliaciones bancarias que no se hace a su debido tiempo, por falta de información en forma oportuna al Auxiliar de Contabilidad; pero sobre todo el personal que labora en este departamento no tiene la suficiente capacitación razón por la cual se incumple con las disposiciones del Reglamento Interno del Municipio y las de Ley Orgánica de la Contraloría General del Estado.

- La falta de aplicación y cumplimiento de la Ley Orgánica de Administración 
Financiera y Control ha dado lugar a que se tenga un ineficiente control sobre el uso y manejo de los bienes; razón por la cual no se tiene procedimientos factibles para determinar las existencias de los bienes obsoletos y comparaciones periódicas de los saldos del mayor en relación con las existencias. El no dar cumplimiento con las disposiciones de la Ley Orgánica de la Contraloría del Estado y el Reglamento interno del Municipio se ha permitido que los activos no se les del tratamiento y conservación que ellos requieren; es así que en su mayoría no se los puede identificar de acuerdo a su código por estar borrado. Esto también ha permitido que se realicen las revalorizaciones de los activos y no se tengan los inventarios actualizados.

\section{Referencias Bibliográficas}

Asamblea Nacional Del Ecuador . (20 de mayo de 2008). Constitución de la República del Ecuador. Obtenido de www.oas.org: https://www.oas.org/juridico/mla/sp/ecu/sp_ecu-int-text-const.pdf

Carmona, M., \& Barrios , Y. (2007). Nuevo paradigma del control interno y su impacto en la gestión pública. Economía y Desarrollo, 141(1), 152-171. Obtenido de https://www.redalyc.org/pdf/4255/425541595009.pdf

Montiel, M., Montiel, C., \& Montiel, Ó. (2017). ¿La implementación del control interno fortalece la gobernabilidad en las alcaldías municipales en Colombia? Administración y desarrollo, 47(1), $97 \quad-117$. Obtenido de file://C:/Users/ENRIQUE\%20GUAM\%C3\%81N/Downloads/DialnetLaImplementacionDelControlInternoFortaleceLaGobern-6403441.pdf

OAS. (15 de mayo de 2013). Ley Organica de Munacipalidad . Obtenido de www.oas.org: https://www.oas.org/juridico/spanish/per_res25.pdf

Prefectura de Pichincha . (17 de Agosto de 2017). Gad Puerto Quito . Obtenido de pichincha.gob.ec: http://www.pichincha.gob.ec/cantones/puerto-quito

Santillan, J. (2015). Sistemas de Control Interno (Tercera edición ed.). México. Obtenido de

http://www.pearsonenespanol.com/docs/librariesprovider5/santillana/978607323

1190_santillana_resolucion_pdf51e66e8b436366b1aea8ff00004a2a88.pdf

Servicios de Rentas Internas . (12 de abril de 2018). Fiscalia Internacional . Obtenido de http://www.sri.gob.ec/web/guest/fiscalidad-internacional2

Sierra , P. (15 de abril de 2015). Diseño e implementación de un manual de control interno para la asociación de ganaderos del Altiplano Cundiboyacense y Occidente De Boyaca "Asogaboy”. Tesis de pregrado Universidad Pedagógica y Tecnológica de Colombia. Bogota. Obtenido de https://repositorio.uptc.edu.co/bitstream/001/2047/1/TGT-658.pdf

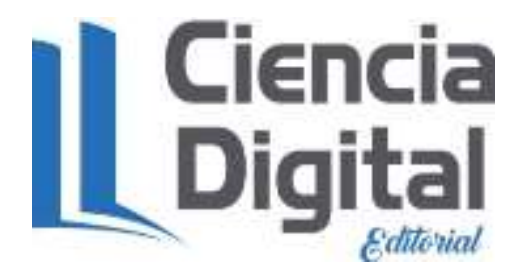




\section{Para citar el artículo indexado.}

Egas F., Cifuentes L., Toala D., \& Vergara Y. (2019) Estudio para la implementación del manual de control interno caso (Gobierno Municipal del Cantón Puerto Quito, Provincia de Pichincha). Revista electrónica Ciencia Digital 3(2), 6 - 26. Recuperado desde: http://cienciadigital.org/revistacienciadigital2/index.php/CienciaDigital/article/view/372 $\underline{1817}$

\section{【Liencia}

El artículo que se publica es de exclusiva responsabilidad de los autores y no necesariamente reflejan el pensamiento de la Revista Ciencia Digital.

El artículo queda en propiedad de la revista y, por tanto, su publicación parcial y/o total en otro medio tiene que ser autorizado por el director de la Revista Ciencia Digital.
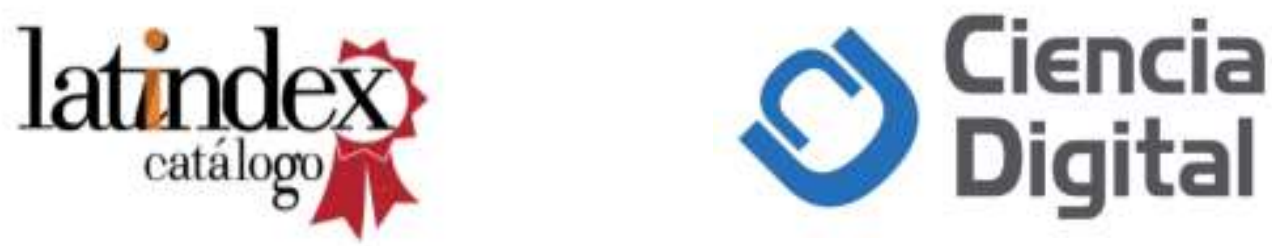